\title{
Educación y jóvenes procedentes del sistema de protección a la infancia
}

\section{Carme Montserrat}

Instituto de Investigaciones sobre Calidad de Vida, Universitat de Girona

<carme.montserrat@udg.edu>

\section{Ferran Casas}

Instituto de Investigaciones sobre Calidad de Vida, Universitat de Girona

Babes-sisteman hartutako haurren eskolatzearen inguruko datuak urriak izaten dira, baina kolektibo honetan gertatzen den hezkuntzarako aukera-desberdintasunek eragin nabarmena omen dute gizarte-bazterketa pairatzeko duten arriskua haztarazteko. Europako azterlan baten baitan, aztertu dira eskolak hartu edo uzteko ematen diren oinarrizko faktoreak babes-sisteman dauden eta aurretik egondako gaztetxoen artean. Berauen ikuspegi propioak biltzen dira eta baita ere gizarte-zerbitzu eta hezitzaileen ardura dutenena. Azterlanaren izaera kualitatibo horrek iradokizunak bideratzeko gaitasuna du praktika profesionala, haurtzaro-politikak eta ikerkuntzan hobekuntzak egiteko.

HITZ-GAKOAK:

babes-sistema, hezkuntzarako aukeradesberdintasuna, babes-sisteman egondako gazteak, haurtzaroa, nerabezaroa.
Son pocos los datos sobre la escolarización de los niños y niñas de los sistemas de protección, pero todo apunta a que la desigualdad de oportunidades en el sistema educativo contribuye en gran medida al alto riesgo de exclusión social de este subconjunto de población. En el marco de un estudio europeo, se han estudiado los principales obstáculos y factores facilitadores que contribuyen a que los niños y niñas en acogimiento residencial o familiar, así como los jóvenes extutelados, continúen sus estudios o bien los abandonen. Se aportan las perspectivas de los propios jóvenes, de responsables de servicios sociales y de educadores sociales. Este estudio de tipo cualitativo permite proponer mejoras en la práctica profesional, las políticas de infancia y la investigación.

\section{Palabras CLAVE:}

sistema de protección, desigualdad de oportunidades educativas, jóvenes extutelados, infancia, adolescencia. 


\section{El proyecto Young People from a Public Care Background (YIPPEE)}

El objetivo general del proyecto 'Young People from a Public care Background: Pathways to Education in Europe' (YIPPEE) fue explorar los itinerarios educativos de los jóvenes extutelados en cinco países de la Unión Europea (Dinamarca, Hungría, España, Suecia y Reino Unido) y estudiar las fórmulas para retener a un mayor número de estos jóvenes en el sistema educativo una vez completada la enseñanza obligatoria. La investigación se llevó a cabo entre 2008 y 2010, fue financiada por la Comisión Europea a través de su VII Programa Marco y se desarrolló de acuerdo con las normas nacionales de ética académica y profesional. En el presente artículo se sintetiza la parte de investigación española ${ }^{1}$-con sus resultados y recomendaciones-, centrada en Cataluña.

\section{Algunos datos de contexto de los sistemas educativo y de protección a la infancia}

En 2007, la tasa neta de la población global que obtuvo el graduado en ESO en España fue del 69,1\%, cifra algo más alta en el caso de Cataluña $(72,8 \%)$. La tasa neta de escolarización a los 17 años era aproximadamente del $75 \%$, con un $11,7 \%$ todavía en la educación obligatoria y un $63,2 \%$ en la educación postobligatoria. Se observaba una mayor presencia de alumnos en cursos académicos que profesionales. Más de un $40 \%$ de la población finalizó la educación secundaria superior (académica, no obligatoria) ${ }^{2}$. En cuanto a la tasa media de abandono escolar prematuro (porcentaje de la población de entre 18 y 24 años que no había completado la educación secundaria postobligatoria y no estaba cursando ningún tipo de ciclo educativo o formativo) se situaba en un $14,8 \%$ para la UE-27. España presentaba una de las tasas más elevadas: $31 \%{ }^{3}$.

Al igual que ocurre en otros países, las tasas de desempleo de la población con cualquier nivel educativo no superior a la primera etapa de la educación secundaria son más elevadas que la tasa media de desempleo. Según los datos de la Encuesta de Población Activa 4 para el primer trimestre de 2008, el desempleo entre la población económicamente activa sin estudios secundarios postobligatorios se situaba en un $24,5 \%$, frente al $5,8 \%$ de la población con estudios de educación secundaria postobligatoria.

Un estudio comparativo internacional de 2004 sobre el gasto público en educación expresado como porcentaje del PIB (Secretaría de Estado de Política

${ }^{1}$ Los informes de los otros países están disponibles en 〈http:// tcru.ioe.ac.uk/yippee/>.

2 Fuente: Ministerio de Educación, Cultura y Deporte («http:// www.educacion.es`).

3 Fuente: Eurostat (〈http://epp.eurostat.ec.europa.eu/portal/ page/portal/eurostat/home/>).

4 Fuente: Eurostat.
Social, 2008) reveló que la inversión de España en materia educativa era menor que en cualquiera de los 27 países de la Unión Europea, con una diferencia del $0,8 \%$ con respecto al promedio de la UE $(4,29 \%$ y $5,09 \%$ respectivamente). Lo mismo es aplicable a la inversión en protección social y políticas familiares, que se sitúa entre las más bajas de Europa: $20,8 \%$ en 2005 , frente al $27,4 \%$ de la UE-25.

Por lo que se refiere a protección a la infancia, el marco legal de Cataluña está anclado en la nueva Ley de los Derechos y las Oportunidades de la Infancia y la Adolescencia, aprobada por el Parlamento de Cataluña el 12 de mayo de 2010. Esta nueva legislación no se centra únicamente en la infancia en situación de alto riesgo social, sino que presenta un enfoque proactivo dirigido a la población infantil y adolescente en su totalidad. Asimismo, proporciona nuevos mecanismos que permiten la participación de niños y adolescentes en los procesos decisorios que les afectan.

Durante los últimos veinte años, desde las comunidades autónomas se ha ido abandonando el modelo 'de beneficencia', caracterizado por grandes instituciones, estancias largas y servicios carentes de coordinación, sustituyéndolo por sistemas de servicios sociales que intenta fomentar el acogimiento familiar como mejor opción dentro del sistema de protección a la infancia. Los cambios en el sistema de protección se basaron inicialmente en el principio de normalización (Casas, 1998), que aboga por mantener a los niños en su entorno familiar y, de no ser posible, proporcionarles un entorno familiar alternativo. Sin embargo, y a pesar de todos los esfuerzos realizados, el hecho de que el $48 \%$ de los niños en acogimiento se hallen aún en centros residenciales refleja las dificultades del sistema para cumplir con este principio de normalización y garantizar el acogimiento en un entorno familiar.

El sistema español de protección a la infancia ha experimentado en los últimos años un aumento en el número de niños en acogimiento. Según estadísticas oficiales ${ }^{5}$, en 2006 en España se contaban 37.161 niños y niñas bajo custodia legal, $74,2 \%$ en tutela administrativa y $25,8 \%$ en guarda administrativa. Los datos desagregados por tipo de acogimiento daban como resultado un $48 \%$ de niños en acogimiento residencial y un $52 \%$ en acogimiento familiar, de los cuales el $15 \%$ estaban en familias ajenas, y el $85 \%$ restante, en familias extensas (Del Valle y Bravo, 2003).

En Cataluña, las estadísticas oficiales de 2007 revelan la existencia de 7.289 niños y niñas bajo alguna medida de protección. En cuanto a las características de los niños en régimen de acogimiento residencial, destaca el peso importante de los niños extranjeros, predominantemente varones mayores de 12 años. Asimismo, se observa que el $6,1 \%$ de los niños y niñas en el sistema de protección a la infancia son menores extranjeros no acompañados, la mayoría de

\footnotetext{
5 Fuente: Ministerio de Educación, Cultura y Deporte.
} 
los cuales se encuentran en régimen de acogimiento residencial. Ante el creciente número de niños que requieren acogimiento, los esfuerzos de la Generalitat de Cataluña no han sido suficientes para impedir la saturación progresiva del sistema, ya que la ocupación en algunos centros supera su capacidad. Una de las razones es la insuficiente oferta de otros recursos, como las familias de acogida.

La mayoría de los centros residenciales presentan una ratio de entre 3 y 4 acogidos por profesional. Las cifras confirman una tendencia cada vez mayor a crear centros residenciales con capacidad para más de 20 chicos/as, lo cual pone en evidencia que la necesidad de acogimiento para una mayor número de niños y adolescentes se soluciona incrementando el número de plazas en los centros, lo que afecta también a la ratio niños-profesionales o la rotación de personal, entre otros aspectos.

Pese al crecimiento del número de familias de acogida que se ha observado recientemente, sigue siendo, en proporción, inferior al aumento del número de niños en centros residenciales y, por lo tanto, insuficiente para cubrir la demanda real. Los acogimientos en familias extensas han aumentado en términos absolutos y constituyen uno de los pilares del sistema de protección a la infancia de España (Montserrat, 2007), que, según un estudio español, muestra mejores resultados en comparación con otros tipos de acogimiento (Del Valle et al., 2008). No obstante, las últimas recomendaciones del Defensor del Pueblo de Cataluña (Síndic de Greuges, 2009) sugerían la necesidad de abordar la falta de una base de datos completa de los niños en acogimiento que también incluyera información sobre procesos y resultados.

Otro aspecto destacable es la gran heterogeneidad respecto a las condiciones de trabajo y a los criterios para elaborar propuestas relativas a las medidas de protección a la infancia que muestran los 47 equipos de atención a la infancia y adolescencia (EAIA) de Cataluña, como consecuencia de una deficiente evaluación del funcionamiento del sistema (Casas y Montserrat, 2002). Además, cabe señalar que el análisis de los presupuestos asignados a los servicios responsables de la protección a la infancia indica que, por cada euro invertido en la familia biológica en 2006, se invirtieron 2 en atención a la familia extensa, 2,5 en la familia de acogida, 24 en centros residenciales y 30 en centros de acogida de urgencias (Síndic de Greuges, 2009).

La información sobre la transición a la vida adulta en Cataluña facilitada por la Secretaría de la Juventud revela que en 2008 tan sólo un 15,8\% de los jóvenes de entre 20 y 24 años llevaban una vida independiente fuera del hogar familiar (el $84,2 \%$ sigue viviendo con los padres) y que el $54,6 \%$ de la población de entre 25 y 29 años todavía no había abandonado el núcleo familiar. En España, la emancipación de los jóvenes se produce a una edad más tardía que en otros países europeos. En cuanto al sistema de protección, pese a que, por ley, la edad máxima para independizarse es 18 años, los jóvenes que viven en familias de acogida ajena o extensa pueden permanecer en ellas si ambas partes (el joven y la familia de acogida) están de acuerdo, aunque sin apoyo económico ni de otro tipo.

El modelo de bienestar español no prevé ayudas de carácter universal que permitan continuar los estudios cuando el joven alcanza los 18 años. Cataluña es una de las pocas comunidades autónomas que cuentan con un servicio de atención a jóvenes extutelados, en el que se ofrecen ventajas para el acceso a una vivienda, a la formación, la inserción laboral o ayudas económicas. El Àrea de Suport al Jove Tutelat i Extutelat (ASJTET) empezó a funcionar hace quince años, mediante acuerdos con distintas ONG para la creación de programas de apoyo a los jóvenes extutelados de entre 16 y 21 años, especialmente a aquellos procedentes de un acogimiento residencial.

\section{Punto de partida al inicio del proyecto YIPPEE}

Los datos expuestos en el punto anterior fueron los que contextualizaron el proyecto iniciado en 2008 , pero no se encontró ninguna información cuantitativa sobre la escolarización de los niños y niñas tutelados, como tampoco sobre los jóvenes extutelados en España. Esta invisibilidad estadística de los jóvenes en el sistema de protección y, en especial, todo lo referente a su situación escolar en España venía acompañada también de una falta de investigación en este campo (Casas y Montserrat, 2012; Montserrat y Casas, 2010; Inglés, 2005).

Uno de los objetivos iniciales del proyecto YIPPEE era "establecer una línea base de la participación en la educación postobligatoria entre los jóvenes extutelados". Sin embargo, no se halló dato estadístico sistemático alguno que ilustrara la participación de estos jóvenes en la educación postobligatoria o superior, ni tampoco acerca de sus logros en la educación secundaria obligatoria. Esta laguna informativa fue el motivo que impidió al equipo de investigación español realizar un análisis secundario de los datos oficiales, proceso que sí que pudieron cumplir Inglaterra, Suecia y Dinamarca. Por consiguiente, el proyecto de estudio se inició con la ausencia absoluta de información sobre la tasa de participación de estos jóvenes en los estudios postobligatorios, ni en España ni en Cataluña.

La revisión de la literatura científica (Montserrat y Casas, 2010) incluyó una síntesis de los pocos estudios disponibles sobre los jóvenes en acogimiento residencial, tanto en familia ajena como extensa; sin embargo, dichos estudios apenas contenían información sobre su educación. El informe del proyecto YIPPEE puede considerarse, por lo tanto, el primer estudio realizado sobre este tema en España. Con el fin de empezar a subsanar esta importante laguna, se acordó con la Generalitat de Cataluña recopilar información durante dos años académicos (20082010), lo que permitiría iniciar la producción de datos 
sistemáticos relativos a los itinerarios y resultados académicos de los alumnos de 16 años en cualquier forma de acogimiento. Los primeros datos ya mostraron que únicamente el $20 \%$ de la población en acogimiento en Cataluña había obtenido el título de graduado en ESO a esa edad, en contraste con el $60 \%$ de los adolescentes de población general (Montserrat, Casas y Bertran, en prensa; Montserrat, Casas y Malo, 2012). Esta iniciativa, no prevista en el proyecto de investigación inicial, ha abierto el camino hacia una medida de acción positiva: prorrogar la recogida de datos hasta 2013.

\section{Metodología de la investigación}

El proyecto se estructuró en tres fases principales:

1. Una revisión de la literatura científica.

2. Un análisis secundario de las estadísticas nacionales sobre la población infantil tutelada y su logro educativo que, en el caso español, se sustituyó por el acuerdo con el Gobierno catalán para iniciar una recogida de datos al respecto. Esta fase incluía también una encuesta a informantes clave, profesionales de administraciones públicas (coordinadores de servicios de atención a jóvenes extutelados, miembros de equipos de protección a la infancia y profesionales de los servicios sociales locales). Con estas entrevistas se perseguía, por un lado, estudiar las opiniones de los entrevistados sobre el proceso de independización del sistema de protección y sobre las opciones de dichos jóvenes en los estudios, y por el otro, acceder a una muestra de jóvenes.

3. Un estudio de casos a partir de entrevistas en profundidad a jóvenes extutelados en cada país, a responsables de los servicios sociales y de protección, y a adultos 'referentes' designados por estos jóvenes.

A través de los servicios de atención, obtuvimos los datos de contacto de una muestra de jóvenes de entre 19 y 21 años que se ajustaban a los criterios de inclusión para el estudio:

- Haber estado en el sistema de protección a la edad de 16 años.

- Haber estado en dicho sistema como mínimo un año.

- Haber mostrado capacidad y motivación para seguir los estudios (por ejemplo haber obtenido el graduado en ESO y mostrar motivación para continuar estudios postobligatorios).

Tras conseguir una muestra de 132 jóvenes que cumplían con estos requisitos y, en 2008 , se realizó una ronda de entrevistas en profundidad con 35 de estos jóvenes, de los cuales 28 fueron entrevistados de nuevo un año más tarde. Una de las peticiones a este grupo de jóvenes fue la que nombraran a un adulto que les hubiera prestado un apoyo especial o clave durante su etapa educativa. Veinte de los adultos designados fueron también entrevistados. Todas las entrevistas se grabaron en audio, se transcribieron y se analizaron con el programa de análisis de datos cualitativos Nvivo. Todo ello, junto con las entrevistas a los profesionales de servicios sociales y de protección, constituyó la base de los resultados cualitativos.

En la primera entrevista con cada joven, se adoptó un enfoque de historia de vida, libremente estructurada por cada entrevistado. No obstante, el entrevistador llevaba una lista de temas que debían abordarse, y que utilizaba si la información no emergía espontáneamente. Asimismo, hacia el final de la entrevista se pedía una 'línea de vida', que sirvió de ayuda a los investigadores para comprender la trayectoria educativa y vital de cada entrevistado. La segunda entrevista fue semiestructurada, así como lo fueron las de los profesionales y adultos de referencia.

Al comparar las características de los 35 jóvenes entrevistados con el conjunto la población tutelada en Cataluña, se observa que:

- No hay diferencias por lo que se refiere a los problemas con la familia de origen, el promedio de años en acogimiento, el año de admisión o el número de acogimientos.

- En la muestra hay un porcentaje más alto de mujeres, posiblemente atribuible al criterio de selección, que requería mostrar motivación para seguir estudiando.

- Predominan los jóvenes que habían estado en centros residenciales, posiblemente debido a que una parte importante de los datos de contacto se obtuvieron del servicio de atención a jóvenes extutelados, que atienden mayoritariamente a los de centros.

- Incluye un porcentaje más alto de menores extranjeros no acompañados y de extranjeros en general.

- Hay una gran diferencia por lo que se refiere a la vuelta con la familia de origen tras alcanzar la mayoría de edad (Del Valle, Imaña y de la Herrán, 2003; García Barriocanal y Barrio, 2007). De hecho, ninguno de los jóvenes entrevistados vivía con sus progenitores en el momento de la entrevista.

- Se da un porcentaje más bajo de abandonos escolares prematuros, atribuible al criterio de mostrar motivación para los estudios. De hecho, muchos de ellos pueden estudiar porque reciben apoyo de los servicios de atención a jóvenes extutelados.

\section{Resultados a partir de la perspectiva de algunos profesionales de servicios en contacto con los jóvenes extutelados}

Recogimos las opiniones de profesionales del servicio de atención a jóvenes extutelados (ASJTET), los servicios sociales locales y los equipos de protección a la infancia (EAIA). También entrevistamos a algunos 
educadores sociales de centros residenciales, por tratarse de los adultos 'referentes' que designaron los jóvenes al final de la entrevista.

En cuanto a las responsabilidades, los planes de acción, las obligaciones y los apoyos prestados por los citados servicios, los profesionales entrevistados reconocieron que aquellos jóvenes en proceso de independizarse del sistema de protección a la infancia que no pueden volver con sus familias de origen o prolongar su convivencia con la familia de acogida se suelen derivar a los mencionados servicios de atención a jóvenes tutelados y extutelados. De ello se encargan normalmente los centros residenciales en los que está acogido el joven, o bien los equipos de protección a la infancia, que redactan una propuesta y un plan de trabajo y seguimiento, acordado previamente entre el joven y el servicio.

Estos informantes clave también coincidieron en señalar que los jóvenes extutelados no suelen acudir a los servicios sociales locales y, si lo hacen, es por decisión propia y como cualquier otro ciudadano. En estos casos, la intervención no es sistemática y no existe un protocolo de actuación; los jóvenes reciben asesoramiento en función de cada caso en particular.

En cuanto a las opciones educativas disponibles para los jóvenes tutelados y extutelados, todos los agentes sociales entrevistados reconocieron que padecen una situación de clara desventaja y que suele empezar a trabajar tan pronto alcanzan la edad mínima permitida por ley. Los servicios consultados también manifestaron que resulta más fácil ofrecer recursos formativos no reglados (sin continuidad en el sistema de educación reglada) a estos jóvenes, puesto que este tipo de recursos presentan menores dificultades de adaptación. Añadieron que es difícil retenerlos en el instituto, debido en parte a la rigidez del sistema, que afecta no solamente a la población de protección, sino también a la que se encuentra con dificultades sociales y familiares en general.

Algunos informantes también afirmaban que, para estos jóvenes, las dificultades ya aparecen en el sistema educativo obligatorio, especialmente en la escuela secundaria: los problemas en sus entornos familiares, las situaciones de inestabilidad y los cambios de escuela, las carencias acumuladas y las etiquetas sociales son factores, todos ellos, asociados al fracaso. Asimismo, reconocen que en teoría no existe ningún problema, pero que en la práctica únicamente unos cuantos continúan estudiando después de los 16 años, y si lo hacen, es en programas de formación profesional de ciclo corto, que les permiten encontrar empleo generalmente en los sectores de la construcción, la restauración o la peluquería.

También, según apuntan los entrevistados, destaca una clara falta de primacía de la educación dentro del sistema de protección, algo que no debería ser así. Las expectativas de los servicios de atención a la infancia en materia de educación son demasiado bajas. Los educadores sociales se preguntaban si el hecho de orientar a los jóvenes fuera del itinerario académico formal está vinculado a las circunstancias económicas, o más bien a la falta de aptitudes y habilidades que los adolescentes en acogimiento parecen mostrar para los estudios. En todo caso, están convencidos de que el tiempo que los jóvenes pasan en el sistema de protección a la infancia mejora sus posibilidades educativas, en comparación con el tiempo que pasan con sus familias. Asimismo, reconocen la resiliencia de algunos de estos jóvenes y creen que son necesarios más servicios de apoyo continuo y de transición.

Con todo, se observa un convencimiento cada vez mayor de la necesidad de ofrecer mejores oportunidades educativas a los jóvenes atendidos por los servicios de apoyo a jóvenes extutelados. Prueba de ello está en Cataluña, donde se han impulsado algunas becas de estudio para los jóvenes extutelados por parte de una entidad bancaria y se ha inaugurado una residencia de estudiantes destinada a este grupo.

\section{Resultados a partir de las experiencias personales de los jóvenes}

\section{1. ¿Cómo era su vida en el momento del estudio?}

En general, los jóvenes entrevistados mostraron satisfacción con el curso de sus vidas y opinaban que les iba mejor que a la mayoría de personas extuteladas. Los aspectos que consideraban más preocupantes eran principalmente los relacionados con la economía y el trabajo, la falta de tiempo para estudiar y las responsabilidades familiares.

En cuanto al nivel educativo más alto alcanzado, cinco cursaban estudios universitarios, doce habían empezado o completado un curso de formación profesional de grado superior, cinco habían empezado o completado la educación secundaria superior, diez habían empezado o completado un curso de formación profesional de grado medio, uno se había quedado sólo con el graduado en ESO y dos, con el certificado de escolaridad.

En la primera entrevista (edad de entre 18 y 21 años), una quinta parte de la muestra había abandonado los estudios y estaba trabajando o buscando empleo. La mitad trabajaba a tiempo completo y algunos habían conseguido compaginar el trabajo con los estudios. El tipo de trabajo que desempeñaban era fundamentalmente no cualificado. Para algunos, el trabajo representaba el éxito personal, mientras que para otros lo era la formación.

Cerca de la mitad de ellos residía en pisos asistidos para extutelados. Los que habían estado en familias de acogida seguían conviviendo con ellas, y el $37 \%$ vivía por cuenta propia. En general, opinaban que su salud era buena, aunque tres de ellos estaban en tratamiento psiquiátrico.

En más de la mitad de los jóvenes, uno o ambos progenitores habían fallecido o se hallaban en 
paradero desconocido. En los casos en que sí que mantenían relación, por lo común no era muy fluida: el joven visitaba o atendía a sus padres, pero éstos no representaban ningún tipo de apoyo para él. A excepción de cuatro de ellos, todos tenían hermanos. Un tercio de los jóvenes asumían algún tipo de responsabilidad hacia sus hermanos menores. Algunos tenían pareja, pero ninguno, hijos; y se sentían bien recibidos por la familia de sus parejas.

Dedicaban el tiempo libre a estar con los amigos y a realizar las mismas actividades que cualquier otra persona de su edad. La responsabilidad que suponía hacer frente a las obligaciones domésticas, cuidar de su familia de origen y compaginar el trabajo con los estudios les hacía sentir distintos de los demás jóvenes de su edad. Con todo, este sentimiento desaparecía cuando estaban con los amigos o la pareja y en su tiempo de ocio, situaciones en las que se sentían uno más, excepto quizá por el hecho de disponer de menos tiempo libre.

\section{2. ¿Cómo les fue con la familia y en el sistema de protección?}

Treinta de los treinta y cinco jóvenes entrevistados fueron víctimas de algún tipo de negligencia o abuso durante el periodo que vivieron con sus padres. Los cinco restantes eran menores extranjeros no acompañados cuyo propósito al llegar a España era encontrar trabajo. Las situaciones de abuso coincidían con la adicción a las drogas o el alcohol de los progenitores, los trastornos mentales, el ingreso en prisión, o la muerte 0 el abandono de éstos. Al hablar de la etapa vivida con sus progenitores, los jóvenes destacaban la gran inestabilidad y los cambios de residencia, y las consecuencias negativas que, a veces, ello comportaba para su estado de salud. En cuanto a la forma en que habían sido educados, los jóvenes extranjeros señalaron grandes diferencias culturales, y los que habían convivido con sus abuelos, diferencias generacionales.

Muchos de ellos evocaban con desagrado las visitas familiares durante el acogimiento, que recordaban de carácter obligatorio. En otros casos, se había producido el abandono de los padres al entrar en acogimiento, y muy pocos conservaban buenos recuerdos de estas visitas. Por lo general, la relación con los hermanos era buena, con la carga adicional que suponía su responsabilidad, especialmente antes de entrar en acogimiento. La separación de los hermanos generaba, sin embargo, sentimientos negativos. Aquellos con familias extensas informaban que, en general, se llevaban bien con sus miembros y que representaban una referencia de apoyo.

En muy pocos casos, las familias de origen habían expresado el valor de la educación, transmitiendo a sus hijos el mensaje de que debían estudiar porque ellos no tuvieron la oportunidad de hacerlo. Por el contrario, la actitud general de los padres era conceder poca importancia a la escuela o a los resultados aca- démicos, proporcionar escaso apoyo en los estudios y mostrar poco interés para que asistieran a clase.

La mayoría de los jóvenes entrevistados reconocieron haber vivido de forma traumática la primera vez que entraron en el sistema de protección: sucedió de repente, sin explicación clara y sin solicitar su opinión. Recordaban especialmente las experiencias negativas asociadas a los centros de acogida y urgencia: centros cerrados y con niños extremadamente problemáticos. Otros se quejaron del largo tiempo que tardaron los servicios en intervenir en sus problemas familiares. La mayoría de los entrevistados habían estado en centros residenciales. De los seis que habían vivido principalmente en régimen de acogimiento familiar, la mayoría lo había hecho en familia extensa y juzgaban positiva la experiencia.

Las valoraciones que estos jóvenes hacían de sus experiencias en acogimiento residencial o familiar eran muy diversas. Se mencionaron desde situaciones óptimas a incluso casos de abuso. En general, los jóvenes resaltaron la importancia de mantener una relación referencial, estable y comprometida con un educador o acogedor. Resaltaron que los centros de gran capacidad, con mucho personal y turnos diferentes, dificultaban la creación de relaciones de confianza, al igual que los cambios de centro residencial y de acogimiento. Otro de los aspectos que destacaron es la importancia de contar con buenas condiciones para el estudio: clases de refuerzo en caso necesario, espacio suficiente en un ambiente de paz y tranquilidad, y motivación para continuar los estudios.

Coincidieron en señalar la importancia de hacer amigos fuera del entorno residencial, entre los compañeros de clase, que estudiaban y no eran conflictivos. Valoraban el apoyo y aceptación recibidos por parte de los padres de sus amigos y la importancia de continuar estudiando con el mismo grupo. Algunos manifestaron haber entablado amistad con otros jóvenes del centro residencial; otros hablaron de la naturaleza conflictiva de los niños en los centros de acogida. Abandonar el centro residencial fue considerado una experiencia estresante y la posibilidad de un piso asistido para personas extuteladas obtuvo muy buena recepción.

Las valoraciones que hicieron estos jóvenes de su experiencia de protección fueron generalmente positivas, en comparación con las condiciones vividas con sus familias de origen; sin embargo, cuando se comparaban con la población general, consideraban que sus itinerarios estaban minados de dificultades. En su opinión, se requería una profunda mejora de las relaciones entre ellos, y los equipos de atención a la infancia y psicólogos.

\section{3. ¿Qué explicaban de su escolarización?}

A pesar de que el 90\% de los jóvenes de nuestra muestra obtuvieron el graduado en ESO a la edad esperada (16 años), sus estudios postobligatorios se 
demoraron más tiempo del habitual, acumulando un retraso progresivo, y en última instancia indefinido, hasta el punto de que el $78 \%$ de los que cursaron un programa de formación profesional de grado superior (postobligatoria) lo hicieron mucho más tarde de lo habitual.

Entre los jóvenes entrevistados observamos cuatro itinerarios educativos postobligatorios:

- Aquellos que iniciaron una vía de formación académica o profesional y acumularon retraso debido a la repetición de un curso, pero no cesaron en su propósito de obtener la titulación. Algunos se encontraban en la educación superior en el momento de la segunda ronda de entrevistas; otros seguían intentando obtener una titulación. En general, este grupo había experimentado menos cambios en la vida familiar o en el sistema de protección en la infancia, manifestaban que les habían transmitido el valor de la educación y habían recibido apoyo en los estudios. Otro factor que, al parecer, había contribuido a su éxito educativo era el hecho de tener un adulto de referencia que fue crucial en su vida.

- Aquellos que iniciaron el camino de formación académica, pero no pudieron completarlo y pasaron a un itinerario de formación profesional, en el que se sucedían las repeticiones e interrupciones. Ninguno de ellos había alcanzado todavía la educación superior. Algunos completaron finalmente la formación profesional de grado medio y empezaron a trabajar.

- Aquellos que iniciaron un itinerario académico o profesional y lo abandonaron sin completarlo. Estaban trabajando o buscando empleo en el momento de la segunda entrevista.

- Aquellos que no siguieron ningún itinerario educativo reglado tras finalizar la educación secundaria obligatoria, pero realizaron un curso de formación ocupacional.

En los tres últimos grupos, destaca una trayectoria vital marcada por mayor inestabilidad y menos apoyo para continuar los estudios en la formación reglada.

Por lo tanto, a pesar de mostrar aptitud y motivación para el estudio a la edad de 16 años, estos jóvenes se encontraban en una situación de moratoria formativa, debido a las dificultades que representaba llevar una vida económicamente independiente, emanciparse del sistema y enfrentarse a los problemas familiares que afectaron a su desarrollo personal antes y después de los 16 años. La convivencia con sus progenitores había supuesto una influencia negativa en su etapa escolar, con absentismo escolar, cambios de escuela y falta de apoyo con los deberes. Sin embargo, algunos de los jóvenes entrevistados lograron desarrollar estrategias de adaptación para continuar estudiando, pese a las condiciones adversas.

El periodo que pasaron en protección tuvo un impacto más bien positivo en su escolaridad, lo cual se reflejó en la asistencia regular a clase y más apoyo en sus estudios, aunque se observan diferencias entre los centros residenciales en cuanto al apoyo prestado. En el lado negativo, destacamos las bajas expectativas por parte de los educadores para con estos jóvenes en cuanto a su participación en la educación postobligatoria y la orientación que se les dio exclusivamente enfocada hacia los cursos de formación ocupacional de ciclo corto, con el fin de que alcanzaran una independencia económica a la edad de 18 años. En algunos casos, esta coyuntura les condujo a descartar el itinerario académico reglado.

En general, la relación con los profesores es juzgada positivamente; aprecian la ayuda especial que recibieron de los docentes y no les gusta que los etiqueten. Las relaciones con los compañeros de clase también consideran que fueron buenas en general, un entorno en el que formaron su grupo de amigos, aunque se dieron algunos casos de acoso escolar debido al color de la piel o a los rasgos físicos del joven. Recuerdan con satisfacción haber sido aceptados por las familias de sus amigos. En la escuela, simplemente querían ser un alumno más y ocultar su situación en protección. Algunos destacan la función compensatoria de la escuela, la cual en ciertos casos sirvió de refugio, mientras que en otros se hace referencia a la poca atención recibida teniendo en cuenta su situación.

Resaltan la gran importancia de la escolaridad, que consideran el medio para desarrollarse como personas, evolucionar respecto a sus padres y a los demás niños del centro residencial, y obtener un trabajo cualificado, con ingresos más altos para llevar una vida mejor. En general, en la escuela adoptaban una actitud de autosuficiencia y autoconfianza, y desarrollaban todas las actividades sin pedir ayuda. Se consideraban competentes, aunque reconocían que las cosas eran más fáciles en la educación obligatoria y que todo se complicaba a partir de ese punto. Los adultos designados que fueron entrevistados coincidieron en afirmar que la muestra de jóvenes entrevistados constituía una excepción con respecto a su trayectoria académica.

\section{4. ¿A qué dedicaban su tiempo libre?}

El grupo de amigos de la escuela contribuía a facilitar la inserción social de los jóvenes entrevistados, al igual que las familias de éstos, cuando se realizaban actividades recreativas. Hacer amistades dentro del centro residencial se vivía como una experiencia más estigmatizante y la mayoría de ellos comentó que los educadores sociales fomentaban amistades de fuera del centro. Los amigos todavía representan el principal punto de apoyo para estos jóvenes. El cambio de escuela significaba la pérdida de amigos y una experiencia que los jóvenes consideran especialmente negativa.

Realizaban las mismas actividades recreativas que el resto de la población. Concretamente durante su 
periodo en protección participaron en actividades más formales y algunos asociaron la entrada en el centro residencial con el inicio de actividades de ocio organizadas: deporte, clases de baile o pertenencia a centros de tiempo libre. Algunos de ellos también participaron en actividades extraescolares, como clases de inglés o música, aunque debido al coste económico de éstas, no todos las tuvieron. Resaltaron la función protectora y compensatoria de algunos centros juveniles, como los esplais ${ }^{6}$ y clubes deportivos.

Durante la convivencia con sus familias de origen, el apoyo recibido para participar en actividades de ocio y tiempo libre procedía de profesionales, aunque manifestaron que solían jugar mucho en la calle y no realizaban actividades formales. Durante el periodo que estuvieron en protección, este apoyo procedía de los educadores o acogedores. Los amigos y sus aptitudes personales también fueron factores influyentes en la elección de la actividad. Por otro lado, las dificultades para compaginar las actividades de ocio con el trabajo y los estudios, especialmente al hacerse mayores, así como los problemas económicos, eran factores que obstaculizaban la continuidad de estas actividades.

El entorno de los amigos y los programas de ocio representaban, para estos jóvenes, la oportunidad de experimentar una situación de normalidad y de sentirse como los otros niños de su edad. También constituían una forma de liberarse de la situación en sus hogares, y una puerta a la diversión, el apoyo y al aprendizaje. En algunos centros juveniles podían hacer sus deberes. Además, los estudios se valoraban altamente en la mayoría de estos equipamientos sociales.

\section{5. ¿Cuáles eran sus esperanzas y sueños de futuro?}

Las aspiraciones manifestadas por estos jóvenes se centraba en seguir en la educación postobligatoria, después de completar la educación secundaria obligatoria, para acceder seguidamente a la educación superior. Una vez alcanzada la mayoría de edad, la obtención de un título universitario seguía constituyendo una realidad a corto o medio plazo para aquellos que siguieron el itinerario académico, en contraposición a aquellos que optaron por un itinerario vocacional o abandonaron los estudios, para quienes la posibilidad de acceder a la educación superior se había convertido en una opción lejana y poco factible.

En general, todos anhelaban obtener un trabajo más cualificado, independencia económica y vivienda propia, así como poder disfrutar del tiempo libre y los amigos. También deseaban que sus hermanos menores tuvieran una vida más fácil que la suya. En el caso de tener hijos algún día, su intención era evitar a toda costa que se repitiera una situación como la que ellos habían vivido y ser unos padres protecto-

\footnotetext{
${ }^{6}$ Centros de tiempo libre en Cataluña.
}

res. Sentían la necesidad de demostrar que ellos no serían como sus padres.

Al preguntarles por sus planes de futuro, estos jóvenes reflejan percepción de control interno, aunque eran conscientes de la adversidad que suponían las dificultades económicas y la falta de apoyo familiar. Afirman tener confianza en sí mismos y la mayoría argumenta que, si han logrado llegar a la situación actual, serán capaces de seguir progresado con esfuerzo y motivación. Asimismo, esperan que los esfuerzos realizados en el pasado y en el presente den su fruto en el futuro.

La mayoría consideraron satisfactorio el año trascurrido entre las dos entrevistas: algunos habían continuado sus estudios, otros habían seguido trabajado y el resto se había independizado. Podríamos afirmar, en términos generales, que eran jóvenes con un alto nivel de optimismo, capaces de adaptarse a las situaciones no deseadas en un principio. Sobre todo, tenían el convencimiento de que, en el futuro, una vez desvinculados del sistema de protección, podrían llevar una vida desprovista de la etiqueta 'niño tutelado' o 'persona con dificultades sociales o familiares'.

\subsection{Su mundo interior}

En general, sus vidas han estado sujetas a sentimientos de inseguridad y temor, que todavía no han conseguido superar, por el hecho de verse obligados a tomar sus propias decisiones y a resolver dificultades relacionadas con la familia, los estudios, los amigos, el trabajo y la vivienda. Una realidad desconocida para la mayoría de niños y jóvenes de la población general de esa edad.

Los recuerdos de su infancia o adolescencia en sus familias de origen y de los traslados cuando se encontraban en acogimiento desencadenaban sentimientos de amargura y sufrimiento. Una vez en la edad adulta, algunos decidieron cortar los lazos con sus familias de origen, mientras que otros se creyeron en la obligación moral de ayudar a sus progenitores. Los padres no representaban una fuente de apoyo para ninguno de ellos, y tampoco tenían la sensación de tener una familia a la que recurrir en caso de necesidad. Todos estos sentimientos confluían en un profundo sentimiento de soledad. Dentro del núcleo familiar, se habían visto obligados por necesidad a desarrollar un sentimiento de responsabilidad propia que, en algunos casos, se extendía hacia los hermanos menores. El dilema para muchos de ellos surgía al alcanzar la mayoría de edad, cuando se preguntaban hasta qué punto debían continuar con el ejercicio de esta responsabilidad hacia algunos miembros de sus familias. La mayoría sentían que únicamente podían confiar en sí mismos.

El periodo que estos jóvenes pasaron en el sistema de protección marcó el inicio o la agravación de un proceso de estigmatización social que les colocó la etiqueta de niños 'carenciados' o 'conflictivos', hasta el punto de que ellos mismos se referían a la 
población fuera del sistema como "los normales". Al mismo tiempo, ello supuso un incentivo para luchar contra dicha etiqueta y demostrar que no debían ser objeto de lástima, ni tampoco considerarlos drogadictos o delincuentes, como, decían, se piensa de ellos en demasiadas ocasiones.

Por otra parte, reconocieron que el acogimiento supuso un alivio en sus vidas, puesto que, de haber permanecido con sus familias de origen, habrían fracasado. Por lo tanto, se mostraban agradecidos con los profesionales que contribuyeron a su desarrollo. Otro aspecto que destacaron insistentemente es el recuerdo traumático que conservan de su entrada en el sistema, así como el profundo sentimiento de miedo e inseguridad que les invadió al abandonarlo a los 18 años.

Al recordar su etapa educativa, mencionaban una gran inestabilidad cuando vivían con sus familias y más apoyo cuando estaban en acogimiento. Para muchos, la escuela fue el lugar donde podían llevar una vida normal y participar en las actividades de ocio y tiempo libre y, en el caso de los jóvenes de nuestra muestra, era evidente que representaba el éxito personal en sus vidas, porque habían logrado buenos resultados académicos. También era el entorno en el que habían conocido a la mayoría de sus amigos y, por lo tanto, encontraron mayor apoyo para seguir adelante. Algunos de ellos guardaban un sentimiento agridulce de esta época, porque les recordaba la imposibilidad de llevar a cabo su ambición, los estudios superiores, aunque reconocían sentirse satisfechos y orgullosos de su situación actual y de lo que hacían.

Demostraban autoconfianza, un sentido de responsabilidad muy desarrollado para su edad y firme propósito de no pedir ayuda; se mostraban conscientes de todo ello y, en este sentido, se sentían diferentes del resto de los jóvenes de la población general, pero también de muchos otros jóvenes extutelados. Sin embargo, en cuanto a las culturas juveniles en las que participaban no se sentían diferentes a los jóvenes de su misma edad.

\section{Resultados e implicaciones para el desarrollo de políticas y prácticas}

El objetivo de este proyecto era explorar los itinerarios educativos de los jóvenes tutelados y extutelados, e identificar los factores que facilitaban o impedían su continuación en los estudios. Los 35 jóvenes entrevistados habían estado en protección durante parte de su infancia y adolescencia, y fueron seleccionados para el estudio porque a la edad de 16 años, cuando todavía se encontraban en acogimiento residencial o familiar, mostraban aptitud y motivación para continuar en la educación postobligatoria; este hecho fue confirmado por los propios jóvenes y adultos a los que designaron. Pero ¿qué factores impulsaron su continuación en los estudios? 0 ¿qué circunstancias les condujo a abandonar la educación? Y, sobre todo, ¿qué factores motivaron su retraso en el sistema educativo en comparación con la población general, si a la edad de 16 años mostraban un potencial equivalente al de la población media? Quizás la pregunta clave es ¿qué factores facilitaron que estos jóvenes estuviesen motivados para seguir estudiando, cuando otros en su misma situación fracasaban en la escuela? Éstas son las cuestiones que hemos tratado de dilucidar, con el fin de proporcionar recomendaciones en diferentes ámbitos.

Se han identificado los siguientes factores facilitadores durante el periodo que estos jóvenes pasaban en el sistema de protección:

- Estabilidad en el recurso de protección.

- Permanencia en la misma escuela.

- Presencia de un adulto referencial estable en sus vidas.

- Prioridad dada a sus estudios desde su recurso de protección.

- Alto nivel de implicación del educador o acogedor en los estudios.

- Altas expectativas del educador o acogedor en materia de estudios.

- Transmisión del valor de los estudios por parte del educador o acogedor y los docentes: los estudios formales resultan un factor clave para dejar atrás su situación de dificultad social grave.

- Pertenencia a un grupo de amigos externos al sistema de protección e integrados en el sistema educativo, que actuó como grupo de referencia y de pertenencia del joven, fomentándose de este modo una identificación social positiva y, por consiguiente, neutralizándose en cierto modo de su estigmatización.

- Implicación del centro escolar, la actitud comprensiva y acogedora hacia la población en acogimiento.

- Participación en las actividades de ocio y tiempo libre, como el resto de la población.

- Mantenimiento de las relaciones con los hermanos, si era su deseo.

- Atención y consideración de sus opiniones, especialmente por lo que respecta a:

- Preferencia sobre el colegio para continuar sus estudios, en particular cuando entraban en el sistema de protección.

- Descontento con el lugar en el que residían.

- Relaciones con los psicólogos y profesionales de los equipos de atención a la infancia que les atendían.

El acogimiento en un centro residencial de pocas plazas, o en una familia de acogida, puede favorecer la creación de las condiciones descritas arriba, aunque el tipo de acogimiento por sí sólo no es condición suficiente para lograr dichos objetivos. 
Además, se identificaron los siguientes factores facilitadores una vez fuera del sistema de protección:

- Presencia de un adulto referencial estable al que puedan recurrir.

- Prioridad otorgada a la educación por parte del servicio de apoyo.

- Disponibilidad de orientación educativa que fomente la educación postobligatoria reglada, según las preferencias y aspiraciones de futuro de los jóvenes.

- Alto nivel de implicación y altas expectativas de los profesionales en materia estudios formales.

- Transmisión del valor de los estudios por parte de los profesionales.

- Disponibilidad de un grupo de amigos externos al sistema de protección integrados en el sistema educativo.

- Implicación del centro docente en la prevención del abandono escolar por parte del joven, teniendo en cuenta sus circunstancias de dificultad.

- Existencia de servicios que presten ayuda en materia de vivienda, becas destinadas a continuar estudiando y continuidad en el apoyo personalizado para quienes así lo requieran, con el fin de aliviar el sentimiento de temor e inseguridad que sienten al alcanzar la mayoría de edad sin el apoyo de una familia.

Cada uno de estos factores da pie a formular recomendaciones y a proponer acciones aplicables en diferentes ámbitos: jóvenes extutelados, profesionales de la educación y sistemas de protección, políticos, medios de comunicación, ciudadanos e investigadores.

\section{Recomendaciones y consideraciones}

Los resultados obtenidos del proyecto YIPPEE desarrollado en España conducen a la reflexión preliminar de tres aspectos destacados:

- El sistema de protección a la infancia debe adoptar una nueva actitud en el terreno escolar, para que los estudios ocupen un lugar más central y prioritario en la vida de los jóvenes tutelados y en los procesos de independización del sistema de protección.

- El sistema educativo debe contemplar a estos jóvenes como un grupo con necesidades educativas específicas, teniendo en cuenta sus circunstancias familiares y otras experiencias traumáticas sufridas durante su vida. Esto significa garantizar el apoyo incluso después de la etapa educativa obligatoria.

- Los dos sistemas deben mejorar su coordinación para resolver una grave situación de desigualdad de oportunidades educativas que puede derivar en una dificultad de inserción laboral por su baja cualificación, e incluso en un alto riesgo de exclusión social para los jóvenes procedentes del sistema de protección a la infancia.

De hecho, la población extutelada ya ha sido identificada como uno de los grupos con mayor riesgo de exclusión social, y este riesgo empieza durante el periodo de educación obligatoria. Utilizando estas ideas generales como base, los puntos de partida deberían ser los siguientes:

- Dar prioridad a los estudios formales.

- Aceptar que éste es un grupo con necesidades específicas en materia de apoyo a los estudios.

- Evitar los cambios de acogimiento, centro residencial y escuela, y trabajar de forma intensa y coordinada con el objetivo de aportar estabilidad a sus vidas.

- Intensificar la colaboración entre los departamentos y sus respectivos servicios para abordar estos retos.

- Mejorar las expectativas sobre la infancia en protección de parte de los agentes implicados.

- Desarrollar acciones innovadoras en todos los ámbitos para dar respuesta a los retos que actualmente plantea la población en el sistema de protección a la infancia.

- Retrasar su independencia económica precoz.

\section{Consideraciones finales}

A partir de este estudio, se plantean las siguientes consideraciones:

- Es una prioridad inmediata que la desigualdad de oportunidades educativas de la que es víctima este grupo de la población adquiera visibilidad social y estadística.

- Es necesario disponer de información e indicadores de los países europeos que permitan un estudio comparativo de la situación actual y una monitorización de los avances realizados a partir de la implementación de acciones en cada país. La iniciativa de recoger datos sobre la situación escolar de los niños y niñas que se encuentran en el sistema de protección, prevista hasta 2013, ha abierto el camino hacia una medida de acción positiva. A partir de ahora, hay que procurar que continúe este proceso de recopilación de datos que permitan analizar los retrasos y obstáculos reales que alteran los itinerarios educativos de la población en acogimiento y combatir la desigualdad de oportunidades educativas que sufre este grupo de la población.

- Es preciso hacer uso del conocimiento de los profesionales, los propios jóvenes, los investigadores y otros agentes sociales que intervienen en el proceso de reducción de barreras y maximización de oportunidades para extender los itinerarios educativos que mejoran el acceso al mercado laboral. 
- Se trata de un grupo de la población con más resiliencia y potencial de éxito de lo que comúnmente se cree.

- Por lo general, los jóvenes extutelados requieren un apoyo más intenso que la población general durante más tiempo, puesto que sus itinerarios educativos se encuentran rezagados en comparación con los demás jóvenes de su edad. A menudo ellos se marcan objetivos ambiciosos que tardan mucho más tiempo en lograr que la población general (como el acceso a la educación superior). Se trata de una población cuyo itinerario educativo se ve truncado debido a los múltiples incidentes y experiencias traumáticas que empañan diferentes momentos de sus vidas.
- Necesitan apoyo generalizado para compensar este retraso.

- Es imprescindible cambiar las expectativas asociadas a esta población, que en general son bajas y estereotipadas.

- Es fundamental retrasar los procesos de inserción en el mercado laboral y prestar el apoyo necesario para prolongar el periodo de estudios y equipararlo con el de la población general

- Se requiere una dosis importante de voluntad política para el cambio: urge coordinar el sistema de protección social y el sistema educativo para garantizar que se implementen medidas de acción que aseguren una mayor igualdad de oportunidades educativas. 


\section{Referencias bibliográficas}

ÀREA DE SUPORT AL JOVE TUTELAT I EXTUTELAT (2008): "Estudi sobre els joves residents en recursos de la DGAIA. L'Àrea de Suport als Joves Tutelats i Extutelats (ASJTET), principal recurs de sortida", Butlletí d'Infància, no 21 , págs. 2-7.

CASAS, F. (1998): Infancia: perspectivas psicosociales. Barcelona, Paidós.

CASAS, F.; y MONTSERRAT, C. (2012): “Percepciones de distintos agentes sociales acerca de la educación formal de los chicos y chicas tutelados", Educatio Siglo XXI, vol. 30, nํㅡ 2, págs. 185-206.

- (2002): “Interdisciplinary teams and decisions making in child protection systems: The experience of Catalonia", International Journal of Child \& Family Welfare, nํㅜ 4, págs. 146-158.

CATALUÑA (2010): “Llei 14/2010, del 27 de maig, dels Drets i les Oportunitats en la Infància i L'adolescència", Diari Oficial de la Generalitat de Catalunya, n- 5.641,2-6-2010, págs. 42.475-42.536 [rhttp://portaldogc.gencat. cat/utilsEADOP/PDF/5641/1098861.pdf>].

DEL VALLE, J. F. et al. (2008): El acogimiento familiar en España. Una evaluación de resultados, Madrid, Ministerio de Educación, Política Social y Deporte, Observatorio de la Infancia [〈http:// www.observatoriodelainfancia.msps.es/ documentos/Informe_acogida_familiar.pdf)].

DEL VALLE, J. F.; ÁLVAREZ, E.; y BRAVO, A. (2003): "Evaluación de resultados a largo plazo en acogimiento residencial de protección a la infancia”, Infancia y Aprendizaje, vol. 26, nํ․ 2, págs. 235-249.

DEL VALLE, J. F.; y BRAVO, A. (2003): Situación actual del acogimiento familiar de menores en España, Oviedo: Universidad de Oviedo;
Departamento de Psicología [<http://www. observatoriodelainfancia.msssi.gob.es/ documentos/Informe_acogida_familiar.pdf)].

EURYDICE (2009): "Fichas breves de los sistemas educativos europeos y reformas en curso. España, febrero de 2009", en EURYDICE, National Summary Sheets on Education Systems in Europe and Ongoing Reforms, Bruselas, Comisión Europea [‘http://eacea.ec.europa.eu/education/ eurydice/documents/thematic_reports/ archives/047EN.zip〉].

GARCÍA BARRIOCANAL, C.; IMAÑA, A.; y DE LA HERRÁN, A. (2007): El acogimiento residencial como medida de protección al menor, Madrid, Defensor del Menor en la Comunidad de Madrid.

INGLÉS, A. (dir.) [2005]: Aprendiendo a volar. Estudio para el análisis de los programas europeos Mentor 15 y Ulises dedicados al proceso de socialización de adolescentes y jóvenes tutelados que han alcanzado la mayoría de edad o están cerca de ella, Murcia, Fundación Diagrama.

MONTSERRAT, C. (2007): "Kinship foster care: A study from the perspective of the caregivers, the children and the child welfare workers", Psychology in Spain, vol. 11, ํㅜ 1, págs. 42-52.

MONTSERRAT, C., CASAS, F. y BERTRAN, I (en prensa): "Desigualdad de oportunidades educativas entre los adolescentes en acogimiento residencial y familiar", Infancia y Aprendizaje.

MONTSERRAT, C.; CASAS, F.; y MALO, S. (2012): “Delayed educational pathways and risk of social exclusion: The case of young people from public care in Spain", European Journal of Social Work [rhttp://dx.doi.org/10.1080/13691457.2012.7 22981)]. 
MONTSERRAT, C. et al. (2011): Los itinerarios educativos de los jóvenes ex-tutelados, Madrid, Ministerio de Sanidad, Política Social e Igualdad.

MONTSERRAT, C.; y CASAS, F. (2010): “Educación y jóvenes ex-tutelados: revisión de la literatura científica española", Educación XX1, vol. 13, nํ2, págs. 117-138 [<http://redalyc. uaemex.mx/src/inicio/ArtPdfRed. jsp?iCve $\left.=70617175005^{>}\right]$.
SECRETARÍA DE ESTADO DE POLÍTICA SOCIAL (2008): “Anexo II. Diagnóstico de situación de la pobreza y la exclusión social en España”, en SECRETARÍA DE ESTADO DE POLIITICA SOCIAL, II Informe Nacional de Estrategias para la Protección Social y la Inclusión Social del Reino de España 2008-2010, Madrid, Ministerio de Educación, Política Social y Deporte.

SÍNDIC DE GREUGES (2009): Extraordinary Ombudsman's Report, Barcelona, Síndic de Greuges.

\section{Agradecimientos}

A la Comisión Europea, por la financiación del proyecto YIPPEE, cuyo equipo español ha sido el Equip de Recerca sobre Infants, Adolescents, Drets dels Infants i la seva Qualitat de Vida (ERIDIQV) de la Universidad de Girona (〈http://www.udg. edu/eridiqv>). Queremos dar las gracias a sus miembros Sara Malo, Irma Bertran, Mònica Gonzalez y Dolors Navarro por su decidido apoyo al equipo. Asimismo, deseamos expresar nuestro agradecimiento a Leticia Araujo y a Manuel VidalRibas, por su colaboración, y a Barney Griffiths, por su trabajo de traducción. Agradecemos en especial el apoyo recibido de la Secretaría de la Infancia de la Generalitat de Cataluña y, en particular, a Jordi Bach. Y, sobre todo, queremos dirigir nuestro más profundo y sincero agradecimiento a los 35 jóvenes entrevistados, a los coordinadores de los servicios sociales, a los educadores y acogedores, a las ONG y a otras tantas personas por la dedicación, compresión y consideración que han mostrado en el tratamiento de este tema tan importante, y que han hecho posible este estudio. Sin la voluntad y el interés de todos ellos, este proyecto no se hubiera realizado. 